\title{
Using Temperature Fluctuation Measurements to Estimate Meteorological Inputs for Modelling Dispersion During Convective Conditions in Urban Areas
}

\author{
Wenjun Qian · Marko Princevac • Akula Venkatram
}

Received: 30 April 2009 / Accepted: 16 February 2010 / Published online: 6 March 2010

(C) The Author(s) 2010. This article is published with open access at Springerlink.com

\begin{abstract}
We examine the performance of several methods to estimate meteorological inputs for modelling dispersion in urban areas during convective conditions. Sensible heat flux, surface friction velocity and turbulent velocities are estimated from measurements of mean wind speed and the standard deviation of temperature fluctuations at a single level on a tower at two suburban sites and at one urban site in Riverside, California. These estimates are compared with observations made at these sites during a field study conducted in 2007 . The sensible heat flux is overestimated in the urban area, while it is underestimated at a suburban site when temperature fluctuations are used in the free convection formulation to estimate heat flux. The bias in heat flux estimates can be reduced through a correction that depends on stability. It turns out that the bias in heat flux estimates has a minor effect on the prediction of surface friction velocity and turbulent velocities. Estimates of sensible heat flux, surface friction velocity and turbulent velocities are sensitive to estimates of aerodynamic roughness length, and we suggest estimating the aerodynamic roughness length through detailed micrometeorological measurements made during a limited field study. An examination of the impact of the uncertainty in estimating surface micrometeorology on concentrations indicates that, at small distances from a surface release, ground-level concentrations computed using estimates of heat flux and surface friction compare well with the those based on observed values: the bias is small and the $95 \%$ confidence interval of the ratio of the two concentrations is 1.7. However, at distances much larger than the Obukhov length, this confidence interval is close to 2.3 because errors in both friction velocity and heat flux affect plume spread. Finally, we show that using measurements of temperature fluctuations in estimating heat flux is an improvement on that based on the surface energy balance, even when net radiation measurements are available.
\end{abstract}

Keywords Boundary-layer parameterizations - Dispersion modelling · Heat flux · Temperature fluctuations · Urban dispersion · Urban meteorology

W. Qian $(\varangle) \cdot$ M. Princevac · A. Venkatram

Department of Mechanical Engineering, University of California, A343 Bourns Hall, 900 University Ave.,

Riverside, CA, USA

e-mail: wqian@engr.ucr.edu 


\section{Introduction}

This study is motivated by the need for methods to estimate meteorological inputs, such as surface friction velocity and heat flux, required by the current generation of dispersion models such as AERMOD (the American Meteorological Society/Environmental Protection Agency Regulatory Model, Cimorelli et al. 2005). One can, in principle, make relatively simple measurements of mean winds and temperatures on a tower at one or preferably more levels, and derive these parameters using Monin-Obukhov similarity theory (MOST) (van Ulden and Holtslag 1985). However, the application of MOST is generally justified when the surface roughness is relatively uniform upwind of the tower for distances of about 100 times the measurement height (Wieringa 1993). Such idealized conditions are rarely met in practice especially in urban areas where dispersion models still have to be applied. One way of estimating meteorological inputs for urban areas is to model the internal boundary layer that develops when rural flow crosses an urban area. Luhar et al. (2006) used this approach to estimate urban parameters in Basel, Switzerland, from measurements made in relatively uniform upwind rural areas. Although such methods have undergone limited evaluation with observations, they are not yet reliable enough for routine dispersion applications. The more empirically acceptable approach is to derive the meteorological inputs from measurements made close to the location where the dispersion model is applied. Thus, the relevant question addressed in this paper is whether MOST can provide useful estimates even when the location of the measurement tower is far from ideal.

The observations analyzed most probably lie in the roughness sublayer (RSL) of the urban area we are considering. We realize that MOST parameterizations are likely to be valid only in the inertial sublayer (ISL), where the flow can be considered in equilibrium with the underlying rough surface and turbulent fluxes are close to constant with height. The RSL is about 2-5 times the average building height (Raupach et al. 1991), which lies below the inertial sublayer. Wind and temperature profiles have been proposed for the RSL (e.g. Garratt 1980, 1992; Harman and Finnigan 2007), though these profiles are functions of parameters that are dependent on stability and canopy characteristics (Garratt 1980, 1983; Harman and Finnigan 2007), which makes it difficult to apply to practical applications. Furthermore, methods proposed by Rotach (1999) require measurements at the top of the RSL, which might be tens of metres high or might not even exist in an inhomogeneous urban area (Kastner-Klein and Rotach 2004).

There is some evidence that a modified MOST might apply in the RSL. Rotach (1999) found that MOST can be used to describe the wind and temperature profiles in the upper part of RSL if scaling variables such as surface friction velocity $\left(u_{*}\right)$ and the Obukhov length $(L)$ are computed using local values of shear stress and heat flux. Oikawa and Meng (1995) reported good agreement with MOST at 0.77 of the canopy height for a suburban RSL.

As far as we know, Hanna and Chang (1992) is the only study that used MOST to estimate meteorological inputs for modelling dispersion in urban areas. They estimated the sensible heat flux in several urban areas using a surface energy balance proposed by Holtslag and van Ulden (1983). Energy balance methods depend on the parameterization of incoming and outgoing shortwave and longwave radiation, and the partitioning of the net radiation at the ground (between ground heat flux, sensible heat flux and latent heat flux). A common approach to this partitioning is based on assuming that the ratio of the sensible to the latent heat flux, the Bowen ratio, can be estimated from land-use data. Holtslag and van Ulden (1983) suggested a more physically realistic method, the Penman-Monteith approach (Monteith 1981), to account for the variation of Bowen ratio with surface moisture conditions. In urban areas, sensible heat can be absorbed and released from urban canopy 
structures. Several studies (Camuffo and Bernardi 1982; Grimmond et al. 1991; Grimmond and Oke 1999a,b) have suggested models, sometimes referred to as objective hysteresis models (OHM), to predict the non-linear relationship between storage heat flux and net radiation.

Hanna and Chang (1992) found that relative errors in estimating micrometeorological parameters were about $20 \%$, but could be much larger during stable conditions when the surface friction velocity, $u_{*}$, was less than $0.2 \mathrm{~m} \mathrm{~s}^{-1}$. They show that their energy balance method is sensitive to the partitioning of sensible and latent heat fluxes (Bowen ratio), and cloud cover, information that is generally unavailable and/or unreliable.

The questions addressed herein are: can measurements of mean wind speed and temperature fluctuations reduce the uncertainties associated with the energy balance method to estimate surface micrometeorological parameters? How far can we apply MOST in urban areas to estimate meteorological inputs for dispersion models?

The study described here extends earlier studies (Princevac and Venkatram 2007; Venkatram and Princevac 2008) on the performance of methods to estimate the surface friction velocity and turbulent velocities in unstable conditions. These estimates depend on the surface heat flux, which can be estimated with measurements of temperature fluctuations using the free convection relationship proposed by Monin and Yaglom (1971) for $\sigma_{T} / T_{*}$, where $\sigma_{T}$ is the standard deviation of the temperature fluctuations, and the temperature scale, $T_{*}$, is the ratio of the kinematic surface heat flux to the surface friction velocity, i.e. $T_{*} \equiv-\overline{w^{\prime} T^{\prime}} / u_{*}$.

In the current study, we examine methods to improve these estimates using formulations such as that proposed by Tillman (1972), who showed that the free convection estimate could be improved through a function of $\zeta=z / L$, which in turn was related to the skewness of temperature fluctuations. Here $L$ is the Obukhov length and $z$ is the effective distance from the ground obtained by subtracting the zero-plane displacement from the measurement height.

Other investigators have also evaluated this approach for different surface types and stability ranges and proposed different forms for $\sigma_{T} / T_{*}$. Albertson et al. (1995) suggested that $\sigma_{T}$ should be measured above the blending height (i.e. above the roughness wake layer) to apply the free convection approach. Wesely (1988) and Hsieh et al. (1996) showed that the free convection relationship applies over non-uniform surfaces with slight modifications to the constant in the relationship. Weaver (1990) concluded that, if the flux is small or the surface is non-uniform, it is necessary to adjust the $\sigma_{T} / T_{*}$ relationship for land-use type.

The results presented by Lloyd et al. (1991) suggest that Tillman (1972) correction for deviation from free convection is not necessary. On the other hand, De Bruin et al. (1993) confirmed the findings of Tillman (1972) on the usefulness of accounting for shear effects. Most of the previous publications applied the above heat-flux estimation methods for bare soil, grass, shrub or forest. Our study examines the applicability of these methods to sites located in urban areas, where the assumptions that underlie them do not necessarily hold. The current study is similar to that of De Bruin et al. (1993) in that it uses measurements of wind speed and temperature fluctuations. We also examine the impact of the uncertainty in estimating heat flux on modelling concentrations associated with surface releases.

\section{Field Study}

The meteorological data used in this study were obtained at three sites in Riverside County, California, U.S.A. in 2007. The three sites lie along an east-west transect designed to study the evolution of the nighttime boundary layer embedded in the easterly wind as it passed through a suburban site, an urban site, and then onto a downwind suburban site. 
Table 1 Morphological parameters for the three sites and $z_{0}$ and $d_{h}$ based on these parameters

\begin{tabular}{llllll}
\hline Sites & $H_{B}(\mathrm{~m})$ & $\lambda_{p}$ & $\lambda_{f}$ & $z_{0}(\mathrm{~m})$ & $d_{h}(\mathrm{~m})$ \\
\hline US & 4 & 0.15 & 0.03 & 0.12 & 0.36 \\
DS & 4 & NA & NA & $0.02-0.4$ & $0-2.0$ \\
CU & 4 & 0.3 & 0.1 & 0.4 & 1.7 \\
\hline
\end{tabular}

Site US (upwind suburban) is in a desert plain in Moreno Valley, with a residential area to the north and east of the measurement tower. To the west and south of the site is grassland (nearly desert) up to $500 \mathrm{~m}$, with sparse trees and houses further upwind. Site DS (downwind suburban) is on top of a bluff located above the Santa Ana River in suburban Riverside, and is surrounded by a mixture of bushes, grasses and sparse trees. Residential areas are at least $1 \mathrm{~km}$ away, although there is one building to the west of the measurement tower (the distance between the building and the tower is about $20 \mathrm{~m}$, and the height, width and length of the building are about 4,15 and $15 \mathrm{~m}$, respectively). As indicated later, this building might play an important role in determining the aerodynamic roughness length for the DS site. Site $\mathrm{CU}$ (centre urban) is located on the street corner of Arlington and Brockton in downtown Riverside, and is surrounded by low-rise buildings that do not vary much in height for all directions up to $2 \mathrm{~km}$ distant. Sites US and CU are $18 \mathrm{~km}$ apart and sites CU and DS are $9 \mathrm{~km}$ apart.

All three measurement sites are in relatively open areas surrounded by buildings and trees. Using Google maps, we used information within a $2 \mathrm{~km}$ radius of the measurement site to estimate the average building height $\left(H_{B}\right)$, the plan area fraction, $\lambda_{p}$, and the frontal area fraction, $\lambda_{f}$, as listed in Table 1 . These parameters have been converted into aerodynamic roughness length and zero-plane displacement, $z_{0}$ and $d_{h}$, using formulations proposed by Grimmond and Oke (1999a,b). Because there is only one building close to the DS site, $\lambda_{p}$ and $\lambda_{f}$ cannot be calculated for this site.

We realize that these estimates of the aerodynamic roughness length and zero-plane displacement have relevance to the calculation of micrometeorological variables only if the measurements and the associated site meet criteria for the applicability of MOST. In our case, these estimates are only meant to provide bounds on the values of $z_{0}$ and $d_{h}$ obtained by fitting MOST profiles to the observed wind speeds during near-neutral conditions. This fitting process is described in a later section.

Each site was equipped with a 3-m tower instrumented with, (1) a sonic anemometer (CSAT3, Campbell Sci.), (2) two soil heat-flux plates (HFP01SC-L Hukseflux), (3) an infrared thermometer (IRTS-P Apogee), (4) a krypton hygrometer (KH20, Campbell Sci.), (5) two soil temperature probes (TCAV-L, Campbell Sci.), (6) a water content reflectometer (CS616L, Campbell Sci.), (7) two air temperature sensors (109- L, Campbell Sci.), and (8) site US had a net radiometer (CNR1, Kipp \& Zonen). The sampling rate for the sonic anemometers is $10 \mathrm{~Hz}$. During post processing we performed data unification with additional control where all data lines flagged as suspicious (diagnostic warning flag is high) are removed (this happened in a negligibly small number of cases, i.e. $<0.1 \%$ ). Delays were introduced into sonic and hygrometer signals to ensure that all the measurements were synchronous. All the cross products are rotated into natural wind coordinates in post processing, as described in Kaimal and Finnigan (1994).

Data were collected from early February through to late April 2007 at Site CU; sites US and DS were operated for shorter periods of time during mid-March through to late April 
2007, and late March to the end of April 2007, respectively. The analysis that follows is based on 1-h averaged data from the sonic anemometers. For rainy conditions, some of the anemometer measurements of shear stress and sensible heat flux were unreasonably high, and after excluding such conditions, there are 526, 577 and $670 \mathrm{~h}$ of data for the US, DS and CU site respectively, within which we analyze 179,215 and $247 \mathrm{~h}$ corresponding to daytime (0900-1700) unstable conditions. The stability $(z / L)$ range is $-4 \times 10^{-4}$ to -18 for the US site, $-3 \times 10^{-4}$ to -7 for the DS site and $-10^{-3}$ to -3 for the CU site. We determined to exclude the nighttime unstable conditions in our study because stable periods intermittently mix with unstable periods, which deteriorates the performance of methods suitable for unstable conditions only. The measurement height is $3 \mathrm{~m}$ for all sites.

A detailed examination of the wind directions corresponding to daytime unstable conditions shows the flux footprint of each site. For the US site, the wind direction covers a wide range from 150 to $360^{\circ}$, which suggests that the land-use footprint for the US site is characteristic of grassland. For the DS site, the prevailing wind sector is from 230 to $360^{\circ}$, and the secondary wind sector is from 000 to $060^{\circ}$, which occurs about $15 \%$ of the time. The flow at the DS site is mostly influenced by a nearby building; bushes, grass, and sparse trees have a secondary impact on the flow. For the CU site, the wind direction is mostly within $260-360^{\circ}$, and since the CU site is surrounded by buildings in all directions, the footprint of the flow at the CU site is considered to be characteristic of urban land use in cities located in the United States. However, the site is not typical of the built-up downtown areas of large cities, which are often dominated by skyscrapers located within a ten block area (e.g. New York City).

Here we do not examine the relationships between measurements made at these different sites, but focus on methods for estimating micrometeorological variables using routine observations at all sites.

\section{Analysis of Observations}

As a first step, we examined the applicability of MOST to the measurements from the suburban and urban sites, which, in principle, do not meet criteria for horizontal homogeneity.

The performance of the models considered here can be described using a variety of statistics, described in Chang and Hanna (2004). We have chosen to use the geometric mean $\left(m_{g}\right)$ and the standard deviation $\left(s_{g}\right)$ of the ratios of the observed to modelled variable as the primary measure of model performance because they can be readily interpreted (Venkatram 2008). They are defined as:

$$
\begin{aligned}
m_{g} & =\exp \left(\left\langle\varepsilon_{m}\right\rangle\right), \\
s_{g} & =\exp \left(\sigma\left(\varepsilon_{m}\right)\right),
\end{aligned}
$$

where \langle\rangle and $\sigma$ represent mean and standard deviation respectively, and $\varepsilon_{m}$ is the residual between the logarithms of model estimate and observation,

$$
\varepsilon_{m}=\ln \left(C_{p}\right)-\ln \left(C_{o}\right),
$$

where $C_{o}$ and $C_{p}$ are observed values and corresponding estimates respectively. The angle brackets refer to an average. The deviation of the geometric mean, $m_{g}$, from unity indicates whether the model is underpredicting or overpredicting, and is a measure of the bias of the model estimate. The geometric standard deviation, $s_{g}$, is a measure of the uncertainty in 
the model prediction with $s_{g}^{2}$ being approximately the $95 \%$ confidence interval for the ratio, $C_{p} / C_{o}$.

The calculation of the geometric mean, $m_{g}$, and the geometric standard deviation, $s_{g}$, using Eq. 1 poses a problem when the observation is close to zero and the corresponding model estimate is finite; the large logarithm of the ratio dominates the calculation. This is avoided by equating $m_{g}$ to the median of the ratio of the observed to predicted concentration ratios, and using the interquartile range of the ratios to estimate $s_{g}$.

\subsection{Surface Friction Velocity}

The surface friction velocity is estimated from the mean wind speed and heat flux measured at a single tower level using the MOST profile (Businger 1973),

$$
U(z)=\frac{u_{*}}{\kappa}\left[\ln \left(\frac{z_{r}-d_{h}}{z_{0}}\right)-\psi_{m}\left(\zeta_{1}\right)+\psi_{m}\left(\zeta_{0}\right)\right],
$$

where $z_{r}$ is the height above the surface, $d_{h}$ is the zero-plane displacement, $z_{0}$ is the aerodynamic roughness length, $\kappa$ is the von Karman constant $(=0.4), u_{*}$ is the friction velocity, $\zeta_{1}=\left(z_{r}-d_{h}\right) / L, \zeta_{0}=z_{0} / L$, the function $\psi_{m}$ is

$$
\psi_{m}(\zeta)=2 \ln \left(\frac{1+x^{\prime}}{2}\right)+\ln \left(\frac{1+x^{\prime 2}}{2}\right)-2 \tan ^{-1}\left(x^{\prime}\right)+\frac{\pi}{2},
$$

for $L<0$, and where $x^{\prime}=(1-16 \zeta)^{1 / 4}$.

The aerodynamic roughness length, $z_{0}$, and zero-plane displacement, $d_{h}$, for each site are obtained by fitting the observed $u_{*}$ to that estimated from the mean wind speed with MOST, as described in Princevac and Venkatram (2007). Measurements with $L>200 \mathrm{~m}$ and wind speed $>2 \mathrm{~m} \mathrm{~s}^{-1}$ were selected to reduce the effects of stability in estimating $z_{0}$. The zero-plane displacement is taken to be $d_{h}=5 z_{0}$ based on Britter and Hanna (2003).

This approach to estimating $z_{0}$ requires detailed micrometeorological measurements that are not available for routine application of dispersion models, and there would be no need for the type of methods discussed herein if such micrometeorological measurements were available for an extended period at a site. On the other hand, it is clearly feasible to conduct a limited field study at the site of interest to obtain $z_{0}$, which can then be used to estimate micrometeorological variables over the extended period, typically several years, required in regulatory modelling. In principle, we estimate $z_{0}$ using the correlations based on building morphology proposed by Grimmond and Oke (1999a,b). However, as we saw earlier, this approach is difficult to apply in a horizontally inhomogeneous urban area.

We are aware that fetch conditions vary with wind sector, especially for the DS site, resulting in different values of $z_{0}$ and $d_{h}$ for different sectors. For the US site, $z_{0}$ for the wind direction from $000^{\circ}$ to $090^{\circ}$ is $0.14 \mathrm{~m}$, while it is $0.12 \mathrm{~m}$ for the remaining sectors; this is consistent with the existence of buildings in the $000^{\circ}$ to $090^{\circ}$ sector. For the DS site, $z_{0}$ varies from $0.19 \mathrm{~m}$ for wind direction less than $250^{\circ}$ to $0.3 \mathrm{~m}$ for $260^{\circ}$ to $280^{\circ}$ and $0.25 \mathrm{~m}$ for all other directions. The building to the west of the measurement tower determines the large value of $z_{0}$ for that sector. For the CU site, the variation in $z_{0}$ is relatively small: $0.31 \mathrm{~m}$ for wind directions less than $240^{\circ}, 0.35 \mathrm{~m}$ for $240^{\circ}$ to $290^{\circ}$ and $0.29 \mathrm{~m}$ for larger than $290^{\circ}$. If we do not consider different fetch conditions for different sectors, only one value of $z_{0}$ is obtained for each site, and we then find that $z_{0}$ is $0.13,0.27$ and $0.31 \mathrm{~m}$ for US, DS and CU sites, respectively.

Note that $z_{0}$ and $d_{h}$ values obtained here for the US and CU sites are consistent with those estimated from morphological parameters (Table 1), although this result could be fortuitous. 


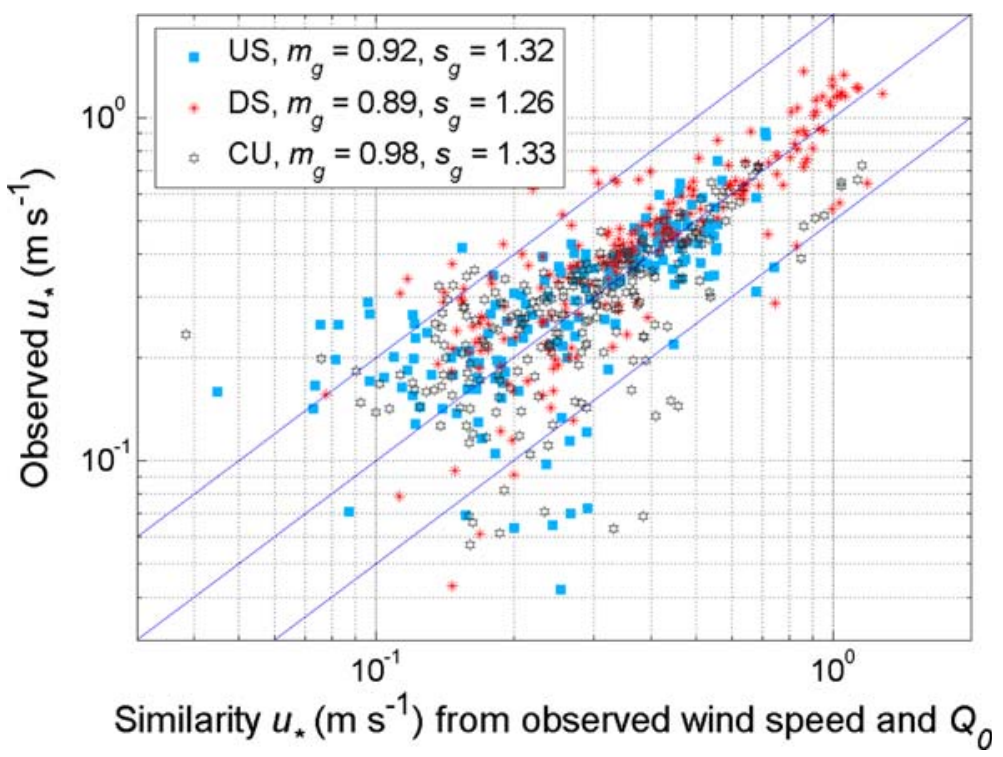

Fig. 1 Comparison of $u_{*}$ estimated from the observed wind speed and heat flux using MOST, with $z_{0}$ and $d_{h}$ obtained for different sectors with observations for the US site (solid squares), DS site (stars), and CU site (open hexagrams)

Routine measurements used in dispersion applications are not likely to include $u_{*}$ and $L$ used to estimate the aerodynamic roughness length. Thus, estimates of the aerodynamic roughness length in an inhomogeneous urban area are likely to be uncertain, and it is useful to examine the impact of this uncertainty on estimating the surface friction velocity, $u_{*}$.

The surface friction velocity, $u_{*}$, is estimated from the observed heat flux, $Q_{0}$, and the wind speed using the approximation of MOST suggested by Wang and Chen (1980) to avoid an iterative solution of Eq. 3,

$$
u_{*}=\kappa u \frac{1+d_{1} \ln \left(1+d_{2} d_{3}\right)}{\ln \left(1 / r_{h}\right)},
$$

where

$$
\begin{aligned}
r_{h} & =\frac{z_{0}}{z_{r}-d_{h}}, \\
d_{1} & = \begin{cases}0.128+0.005 \ln \left(r_{h}\right), & \text { for } r_{h} \leq 0 \\
0.107, & \text { otherwise }\end{cases} \\
d_{2} & =1.95+32.6 r_{h}^{0.45}, \\
d_{3} & =\frac{Q_{0} \kappa g\left(z_{r}-d_{h}\right)}{T_{0}\left\{\kappa U / \ln \left[\left(z_{r}-d_{h}\right) / z_{o}\right]\right\}^{3}},
\end{aligned}
$$

where $T_{0}$ is the surface temperature and $g$ is the acceleration due to gravity.

The results shown in Fig. 1 are based on $z_{0}$ and $d_{h}$ fitted for different sectors. As expected, the estimates of $u_{*}$ with MOST compare well with observed values for both urban and suburban sites; the values of $m_{g}$ indicate a bias of about $10 \%$. The $95 \%$ confidence interval for the ratio of the observed and estimated $u_{*}$ is about 1.7 , but the scatter is large for $u_{*}$, close to $0.1 \mathrm{~m} \mathrm{~s}^{-1}$. 


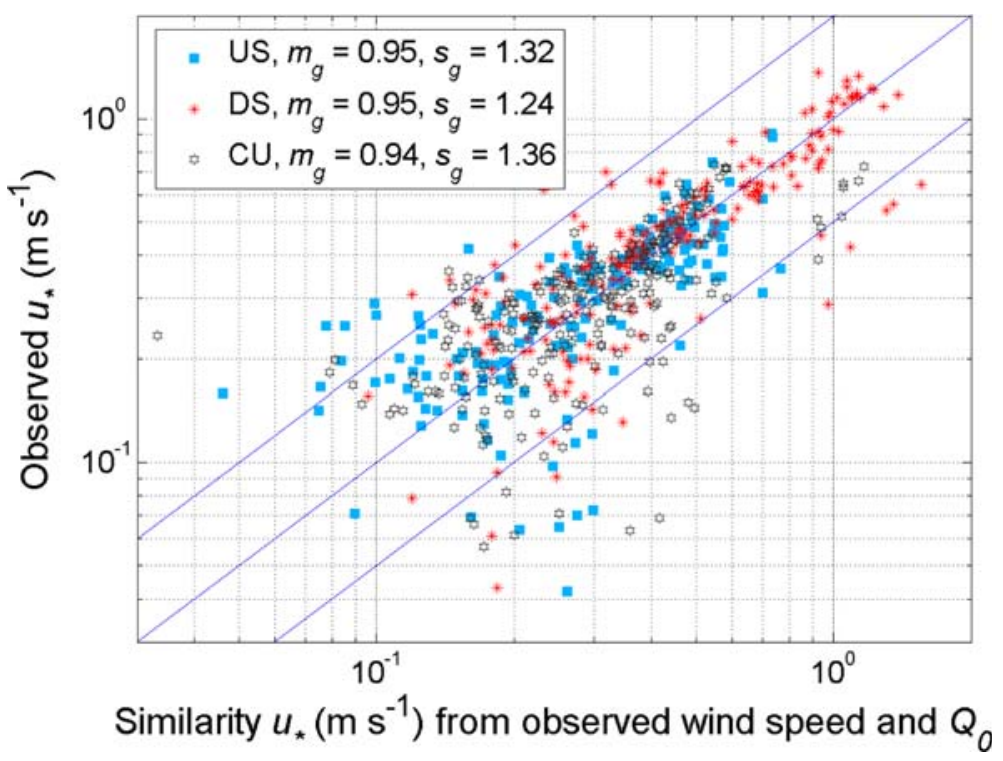

Fig. 2 Comparison of $u_{*}$ estimated from the observed wind speed and heat flux using MOST, with one set of values of $z_{0}$ and $d_{h}$ for each site ( $z_{0}$ is $0.13,0.27$ and $0.31 \mathrm{~m}$ for US, DS and CU sites, respectively) with observations for the US site (solid squares), DS site (stars), and CU site (open hexagrams)

Figure 2 shows results when only one set of values of $z_{0}$ and $d_{h}$ is used for each site, i.e. $z_{0}$ is $0.13,0.27$ and $0.31 \mathrm{~m}$ for US, DS and CU sites, respectively, and $d_{h}=5 z_{0}$. The results are similar to those shown in Fig. 1, although the scatter increases slightly for the CU site: the geometric standard deviation, $s_{g}$, increases from 1.33 to 1.36 .

Figure 3 shows that using half of the values of $z_{0}$ and $d_{h}$ results in an underestimation of $u_{*}$ by $40 \%$ for the CU site to $23 \%$ for the US site. However, most of the model estimates are still within a factor of two of the observations. The $95 \%$ confidence interval for the ratio of the observations and estimates is less than 1.85. Thus underestimating $z_{0}$ and $d_{h}$ appears to yield acceptable estimates of $u_{*}$, but using twice the values of $z_{0}$ and $d_{h}$ leads to unacceptable values of $u_{*}$ (not shown here). The deterioration in our particular case is caused by $z_{0}$ becoming comparable to the effective measurement height, $z_{r}-d_{h}$.

These results indicate that, (1) estimates of surface friction velocity are, as expected, sensitive to the estimate of aerodynamic roughness length, and (2) we might be able to obtain empirical estimates of $z_{0}$ that yield adequate estimates of surface friction velocity even when the area surrounding the measurement site is highly inhomogeneous. In the analysis of the following sections, we use the values of $z_{0}$ and $d_{h}$ that were fitted for different sectors, and we examine the applicability of MOST in estimating the standard deviation of the horizontal and vertical turbulent velocities.

\subsection{Vertical Turbulent Velocity $\left(\sigma_{w}\right)$}

We estimate $\sigma_{w}$ by treating the variable as a combination of a shear-generated component, $\sigma_{w s}$, and a buoyancy-generated component, $\sigma_{w c}$

$$
\sigma_{w}=\left(\sigma_{w s}^{3}+\sigma_{w c}^{3}\right)^{1 / 3}
$$




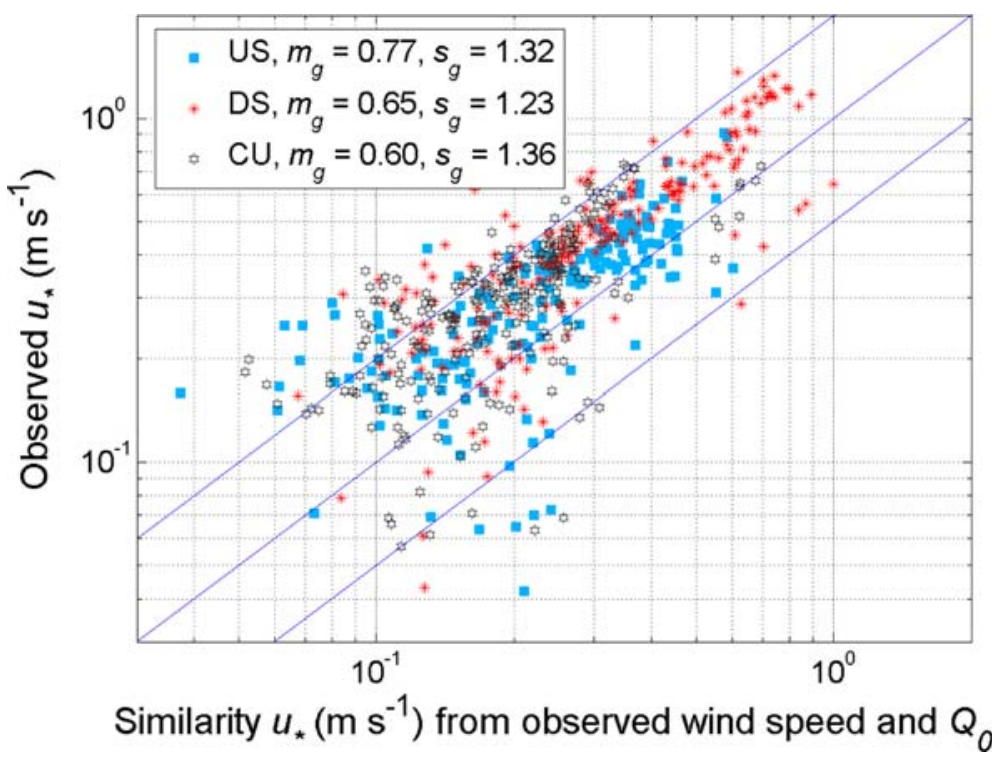

Fig. 3 Comparison of $u_{*}$ estimated from the observed wind speed and heat flux using MOST, with half of the fitted values of $z_{0}$ and $d_{h}$ for each site ( $z_{0}$ is $0.065,0.135$ and $0.155 \mathrm{~m}$ for US, DS and CU sites respectively) with observations for the US site (solid squares), DS site (stars), and CU site (open hexagrams)

where the shear component, $\sigma_{w s}$, is taken to be

$$
\sigma_{w s}=1.3 u_{*}
$$

and the convective component, $\sigma_{w c}$, is

$$
\sigma_{w c}=1.3\left(\frac{g}{T_{0}} Q_{0} z\right)^{1 / 3},
$$

where $z=z_{r}-d_{h}$ is the effective measurement height. Note that $\sigma_{w s}$ and $\sigma_{w c}$ are not added directly in Eq. 7, but rather their cubes are added to ensure consistency with the turbulent kinetic energy equation. Equation 7 can be rearranged to obtain

$$
\sigma_{w}=\sigma_{w s}\left[1+\left(\frac{\sigma_{w c}}{\sigma_{w s}}\right)^{3}\right]^{1 / 3}=1.3 u_{*}\left(1-\frac{z}{\kappa L}\right)^{1 / 3},
$$

where the Obukhov length is defined as:

$$
L=-\frac{T_{0}}{g} \frac{u_{*}^{3}}{\kappa Q_{0}} .
$$

This expression for $\sigma_{w}$ is that presented by Panofsky et al. (1977) to fit a wide range of data. Equation 10 is used to calculate $\sigma_{w}$ using the observed $u_{*}$ and $L$. Figure 4a shows little bias in the estimates, less than $10 \%$, relative to the $\sigma_{w}$ observed at the US and CU sites, but $\sigma_{w}$ is overestimated for the DS site by about $14 \%$. The scatter at all three sites is relatively small with a $95 \%$ confidence interval of about 1.3 .

Previous studies (Clarke et al. 1982; Rotach 1993; Roth 1993; Feigenwinter 2000; Christen 2005) report similar results but have used smaller constants in Eq. 10. 

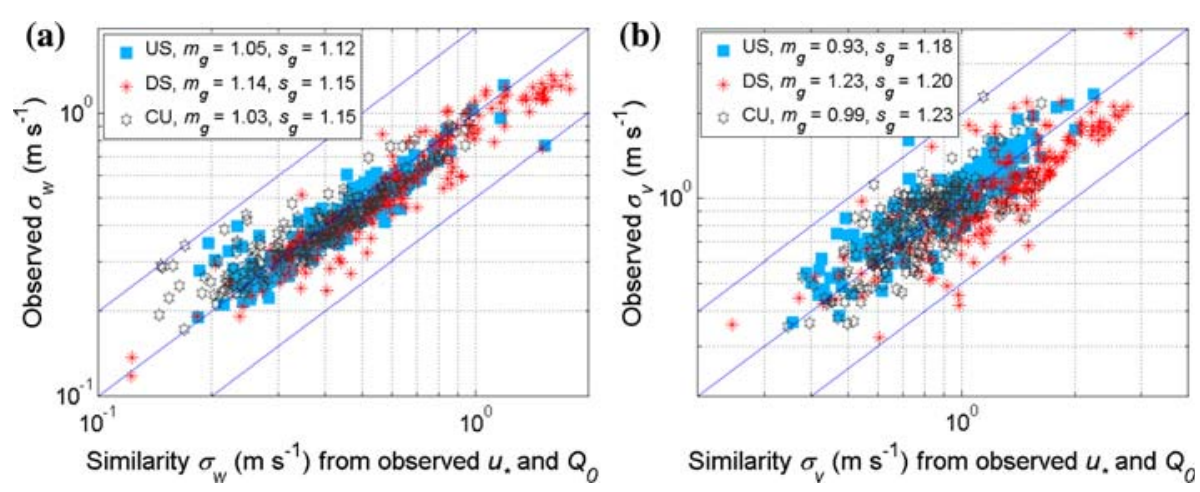

Fig. 4 Comparison of $\mathbf{a} \sigma_{w}$ calculated from the observed $u_{*}$ and $L$ using Eq. 10, and $\mathbf{b} \sigma_{v}$ estimated from the observed $u_{*}$ and $Q_{0}$ using Eqs. 12-14 with observations for the US site (solid squares), DS site (stars), and CU site (open hexagrams)

\subsection{Horizontal Turbulent Velocity $\left(\sigma_{v}\right)$}

The standard deviation of the horizontal velocity fluctuations, $\sigma_{v}$, is computed from

$$
\sigma_{v}=\left(\sigma_{v s}^{3}+\sigma_{v c}^{3}\right)^{1 / 3}
$$

where the shear component is $\sigma_{v s}=1.9 u_{*}$ the convective component is $\sigma_{v c}=0.6 w_{*}$, and the convective velocity scale $w_{*}$ is defined as

$$
w_{*}=\left(g Q_{0} z_{i} / T_{0}\right)^{1 / 3} .
$$

The height of the mixed layer, $z_{i}$, is calculated from a model of a mixed layer eroding a capping layer with a stable potential temperature gradient, $\gamma$ (Carson 1973)

$$
\rho c_{p} \frac{1}{2} \gamma z_{i}^{2}=\int_{0}^{T} H(t) d t,
$$

where $\rho$ is the air density, $c_{p}$ is the heat capacity under constant pressure, $H$ is the heat flux, $t$ is time, and $T$ is a time scale. The unknown potential temperature gradient, $\gamma$, above the mixed layer is taken to be a nominal value of $5 \mathrm{~K}$ per $1,000 \mathrm{~m}$. The sensitivity of the convective velocity to $\gamma$ is relatively small because it is inversely proportional to the $1 / 6$ th power of $\gamma$. Figure $4 \mathrm{~b}$ shows that $\sigma_{v}$ is overestimated at the DS site by about $23 \%$, but the bias is less than $10 \%$ at the other two sites. The $95 \%$ confidence interval of the ratio of the observed to estimated $\sigma_{v}$ is 1.5 .

The results presented here indicate that MOST provides an adequate description of the observations made at suburban and urban sites. These results motivate us to apply MOST to estimate micrometeorological variables using measurements that can be made routinely. Specifically, we focus on methods that use wind speed at one level and the standard deviation of temperature fluctuations, which can be measured using fast response thermistors. 


\section{Temperature Fluctuations Related to Heat Flux}

The heat flux is related to the standard deviations of temperature and vertical velocity fluctuations as follows:

$$
\overline{w^{\prime} T^{\prime}}=r_{w T} \sigma_{w} \sigma_{T},
$$

where $\sigma_{T}$ is the standard deviation of temperature fluctuations $T^{\prime}$, and $\sigma_{w}$ is the standard deviation of the vertical velocity fluctuations $w^{\prime}$.

In this section, we use the data collected at all three sites to examine the behaviour of the correlation coefficient between the velocity and temperature, $r_{w T}$, and then formulate an expression for the heat flux that can be used in routine applications. The objective is to develop methods to estimate heat flux, surface friction velocity, and the standard deviation of vertical velocity fluctuations using only measurements of $\sigma_{T}$ and wind speed at one level. Substituting the expression of $\sigma_{w}$ from Eq. 10 into Eq. 15, and using the definition of the temperature scale, $T_{*}=-\overline{w^{\prime} T^{\prime}} / u_{*}$, yields

$$
\frac{\sigma_{T}}{T_{*}}=-\frac{1}{1.3 r_{w T}}(1-z / \kappa L)^{-1 / 3},
$$

where the correlation coefficient, $r_{w T}$, is a function of $z / L$ in general.

The proposed expression for the correlation coefficient, $r_{w T}$, is based on observations reported in the literature. Monin and Yaglom (1971) indicated that $r_{w T}$ increases from about 0.35 for near-neutral conditions, to about 0.6 for the gradient Richardson number, $R i$, in the range -0.3 to -0.8 . Hicks (1981) also suggested that $r_{w T}$ approaches a constant value, but this requires an unrealistic sign change across neutral conditions. As we will see, the expression presented by Tillman (1972) for $\sigma_{T} / T_{*}$ implies an explicit relation for $r_{w T}$ in terms of $z / L$.

The behaviour of the correlation coefficient in the free convective regime can be derived by equating Monin and Yaglom (1971) expression for the temperature fluctuations,

$$
\frac{\sigma_{T}}{T_{*}}=-C_{1}\left(-\frac{z}{L}\right)^{-1 / 3}
$$

where $C_{1}$ is a constant, to Eq. 16 to yield

$$
r_{w T}=\frac{(-z / L)^{1 / 3}}{1.3 C_{1}(1-z / \kappa L)^{1 / 3}} .
$$

Note that $r_{w T}$ approaches zero as $L$ becomes large for near-neutral conditions. The explicit expression for the heat flux under free convection is

$$
Q_{0}=\left(\frac{\sigma_{T}}{C_{1}}\right)^{3 / 2}\left(\frac{g \kappa z}{T_{0}}\right)^{1 / 2},
$$

while Tillman (1972) semi-empirical correction to Eq. 17

$$
\frac{\sigma_{T}}{T_{*}}=-C_{1}\left(C_{2}-\frac{z}{L}\right)^{-1 / 3}
$$

yields

$$
r_{w T}=\frac{\left(C_{2}-z / L\right)^{1 / 3}}{1.3 C_{1}(1-z / \kappa L)^{1 / 3}}
$$


where $C_{1}=0.95$ and $C_{2}=0.0549$ are the suggested values. Here $r_{w T}$ approaches 0.3 as $L$ becomes large. Note that Eq. 20 results in the following implicit expression for the sensible heat flux:

$$
Q_{0}=u_{*}\left(\frac{\sigma_{T}}{C_{1}}\right)\left(C_{2}-\frac{z}{L}\right)^{1 / 3}
$$

where the value of $Q_{0}$ has to be obtained iteratively because both $u_{*}$ and $L$ are functions of $Q_{0}$.

In the next section, we examine observations of $r_{w T}$ in the light of Eqs. 18 and 20. We also examine the usefulness of a constant value of $r_{w T}$ to explain the observed heat flux using the implicit expression,

$$
Q_{0}=r_{w T} \sigma_{w} \sigma_{T}=r_{w T} \sigma_{T} 1.3 u_{*}\left(1-\frac{z}{\kappa L}\right)^{1 / 3},
$$

which also has to be solved iteratively.

\section{Evaluation with Field Observations}

The left panel of Fig. 5 shows the observed correlation coefficient, $r_{w T}$, as a function of $-z / L$ at the US site compared with the three alternative formulations described in the previous section. The data show that $r_{w T}$ decreases with $-z / L$ but the scatter is large especially for near-neutral conditions. The right panel shows a clear increase of $\sigma_{T} / T_{*}$ with a decrease in $-z / L$. However, we need to be cautious about inferring too much from the data in view of the small values of heat flux at small values of $-z / L$ and the false correlation introduced by non-dimensional variables used (see Hicks 1981 for a discussion).

The coefficient, $C_{1}=0.95$, was suggested by Tillman (1972). However, Wesely (1988) and Hsieh et al. (1996) suggested larger values for $C_{1}$ in the free convection relationship to give results applicable to non-uniform surfaces. We will discuss this issue later in this section. We find that most of the observations of $r_{w T}$ are best described by Tillman's method (Eq. 21) when compared with the other two curves, while the free convection curve (Eq. 18) follows the low values of $r_{w T}$ for near-neutral conditions. Equation 20 provides an adequate description of $\sigma_{T} / T_{*}$ at values of $-z / L$ as low as 0.01 but approaches a constant value at neutral conditions, while the observed data continue to increase. The nominal value of $r_{w T}=0.3$ represents the median of the data; the associated $\sigma_{T} / T_{*}$ simply reflects the variation of $\sigma_{w}$ with $z / L$. The plots of $r_{w T}$ and $\sigma_{T} / T_{*}$ for the DS and CU sites are similar to the results shown here for the US site, and are not shown here.

Since both the free convection formulation and Tillman's correction deviate from the data at low $-z / L$, it is reasonable to examine the utility of a constant $r_{w T}$ in estimating the heat flux and turbulent velocities.

Figure 6a shows the variation of the ratio of heat flux estimated from the free convection formulation (Eq. 19) to the observed heat flux with stability, $-z / L$; the statistics, $m_{g}$ and $s_{g}$, for each site are also listed. As expected, the performance of the free convection formulation improves with an increase in $-z / L$, as shear effects become smaller. The ratios of the estimated to observed heat fluxes show large deviations from unity when $-z / L$ is less than 0.1 , and $m_{g}=0.85$, suggesting an underestimation at the DS site reflects a behaviour at low $-z / L$ that cannot be readily explained. The overestimation of $24 \%$ at the CU site is more consistent with the behaviour of the free convection formulation at low $-z / L$. 

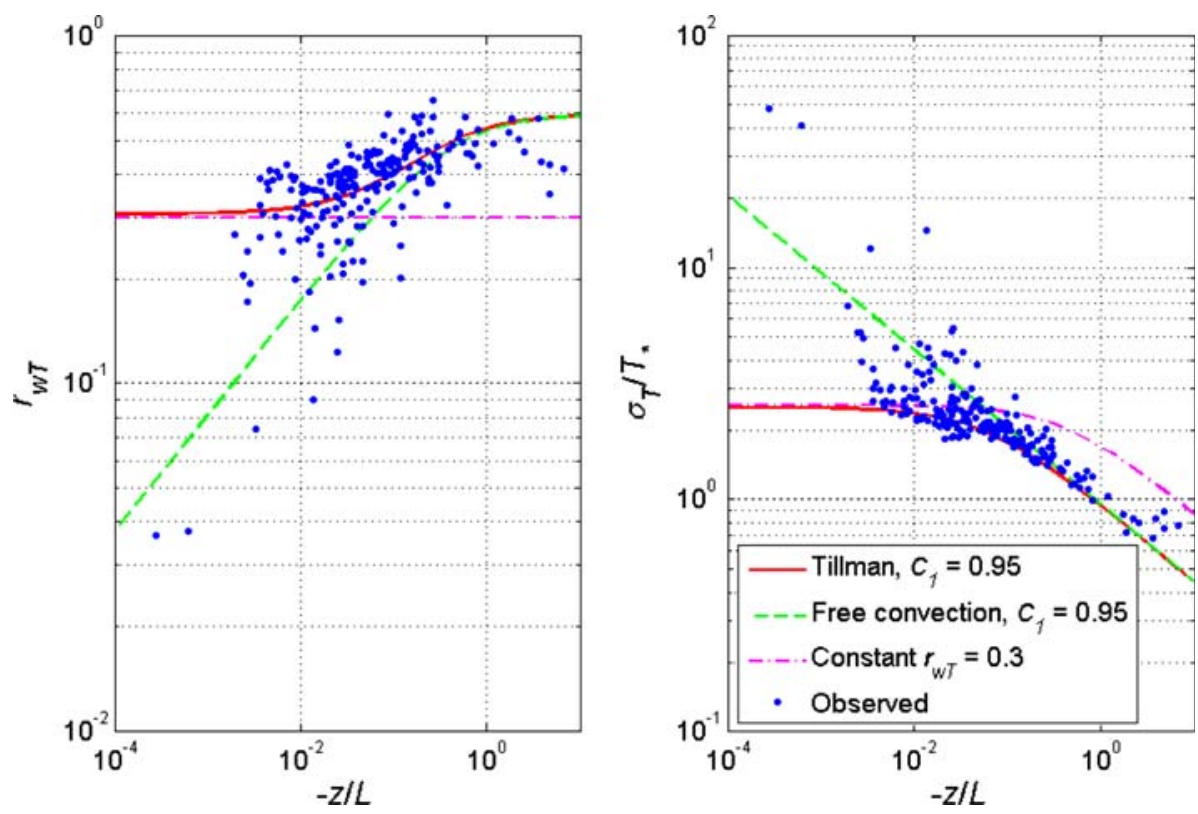

Fig. 5 Comparison of estimates of the correlation coefficient, $r_{w T}$ (left panel) and $\sigma_{T} / T_{*}$ (right panel) as a function of $-z / L$ with observations. The solid line corresponds to Tillman's method (Eq. 20) with $C_{1}=0.95$, the dashed line corresponds to free convection (Eq. 17) with $C_{1}=0.95$, and the dash-dot line corresponds to a constant value of $r_{w T}=0.3$. Measurements were made at the US site

Figure 6b shows the performance of Tillman's method, Eq. 22, as a function of $-z / L$, and we see that the underestimation at low $-z / L$ at the DS site is reduced through the correction for shear incorporated in Tillman's method. However, the heat flux is overestimated by $32 \%$ and $76 \%$ at the US and the CU site respectively. A larger value of $C_{1}$ in the free convection relationship suggested by Wesely (1988) and Hsieh et al. (1996) would decrease the overestimation in the heat flux.

Figure $6 \mathrm{c}$ shows that using constant $r_{w T}$ in Eq. 23 results in an overestimation of heat flux for near-neutral conditions and an underestimation when $-z / L$ is larger. Overall, the heat flux is overestimated by $27 \%$ for the CU site but underestimated by $16 \%$ and $22 \%$ for the US site and DS site respectively. The method has the largest scatter, measured by $s_{g}$, compared to the other two approaches.

These results indicate that in an urban area, estimates of the heat flux that account for stability effects, such as Tillman's, do lead to improvements over the simple free convection estimate at low $-z / L$. Although, the heat flux is overestimated, Tillman's correction has the smallest scatter as measured by $s_{g}$. In the sections that follow, the heat flux is estimated with this method, but $C_{1}$ is taken to be 1.25 , as in Wesely (1988), to reduce the bias.

We next examine the impact of errors in estimating the heat-flux approximations on estimating $u_{*}, \sigma_{w}$ and $\sigma_{v}$. The estimates of $u_{*}$ in Fig. 7a are based on Eq. 22, which requires an estimate of $u_{*}$.

The overestimation of heat flux at the CU site or the underestimation at the DS site has little effect on estimating $u_{*}$, as seen in Fig. 7a. The geometric mean $\left(m_{g}\right)$ and the geometric standard deviation $\left(s_{g}\right)$ are almost identical to those when the observed heat flux is used in Fig. 1. It turns out that $u_{*}$ estimates based on heat-flux estimates from the free convection 

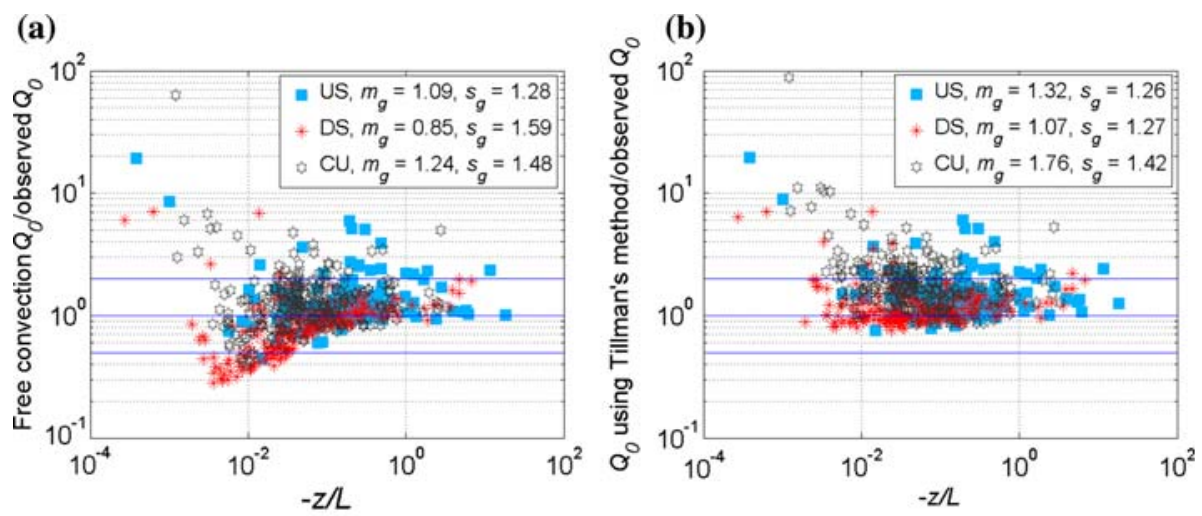

(c)

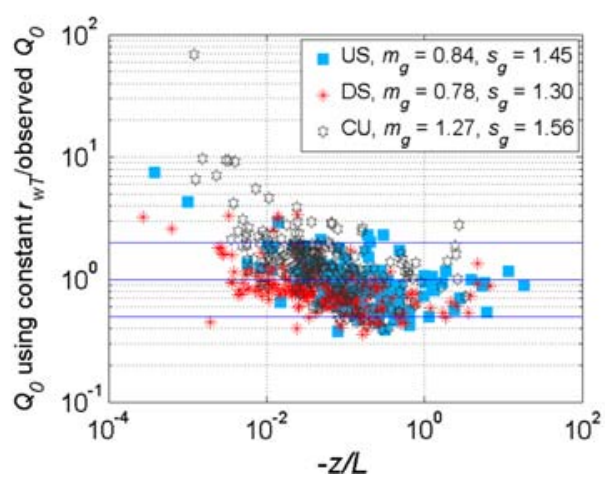

Fig. 6 Ratio of heat-flux estimates from a free convection, Eq. 19, b Tillman's method, Eq. 22, c using constant $r_{w T}$, Eq. 23, to observations as a function of $-z / L$ for the US site (solid squares), DS site (stars), and CU site (open hexagrams)

formulation and the constant $r_{w T}$ approach produce comparable results. This insensitivity of $u_{*}$ to heat-flux errors is related to the fact that $-z / L$ is much smaller than unity (see Fig. 5) for most of the measurements. Figure 7b and c compares estimates of $\sigma_{w}$ and $\sigma_{v}$ with observations from the three sites. We see that the overestimation of heat flux has little impact on estimating $\sigma_{w}$ and $\sigma_{v}$ : there is little bias in the model estimates and the scatter is relatively small. The next question is: how does this uncertainty in estimating micrometeorological variables affect concentration estimates?

\section{Impact on Dispersion Modelling}

Here, we examine the impact of the uncertainty in the estimates of heat flux and friction velocity on modelling ground-level concentrations through the use of the cross-wind integrated ground-level concentration associated with surface releases (Venkatram 1992). This has been evaluated with data from the Prairie Grass experiment (Barad 1958), and is currently incorporated in AERMOD (Cimorelli et al. 2005):

$$
\bar{C}_{*}^{y}=\frac{1}{x_{*}\left(1+\alpha x_{*}^{2}\right)^{1 / 2}},
$$



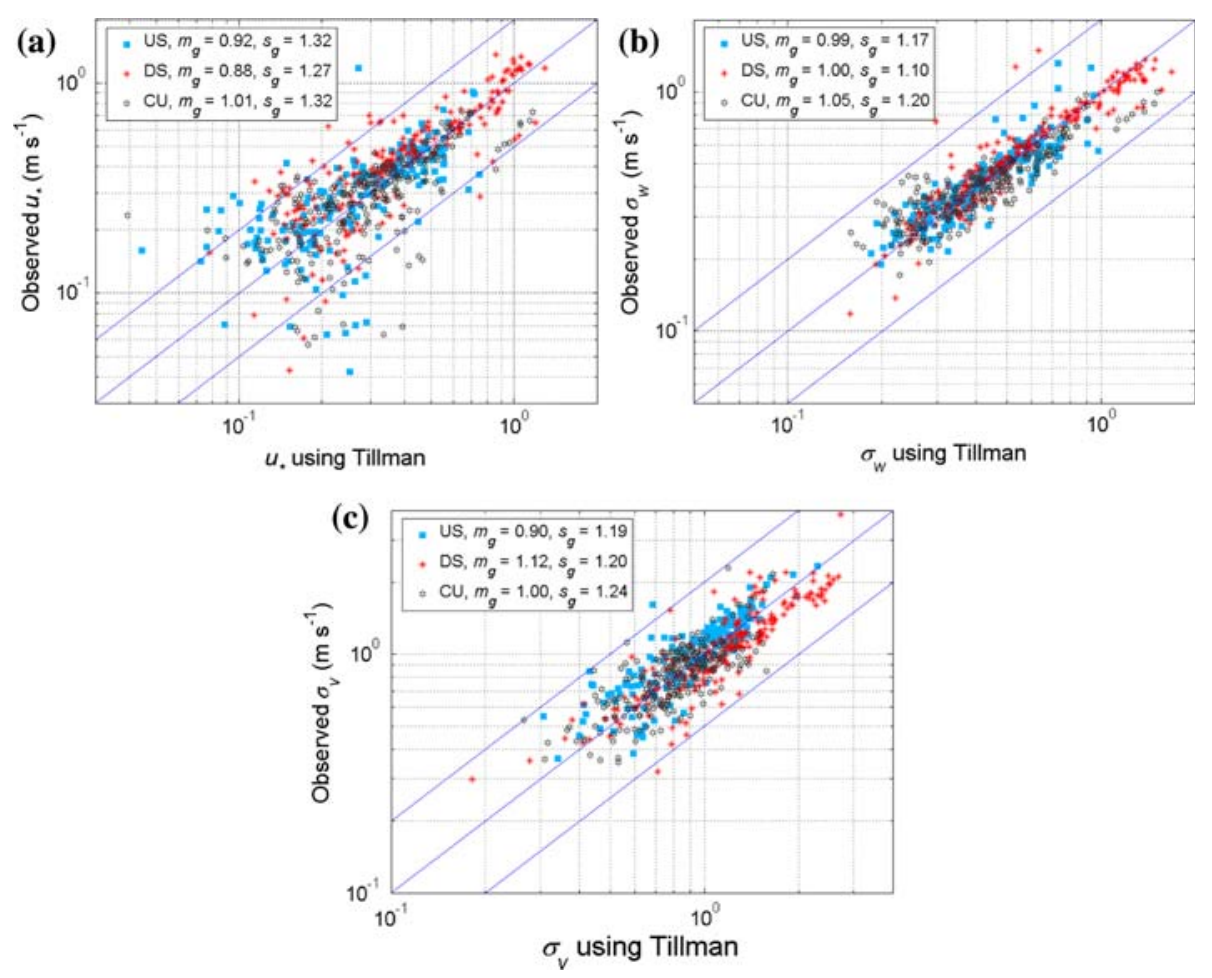

Fig. 7 Comparison of estimated a $u_{*}$ from Eq. 5, $\mathbf{b} \sigma_{w}$ from Eq. 10, and $\mathbf{c} \sigma_{v}$ from Eq. 12 with observations for the US site (solid squares), DS site (stars), and CU site (open hexagrams). The heat flux is estimated from Eq. 22

where $\alpha=6.0 \times 10^{-3}, \bar{C}_{*}^{y}=\bar{C}^{y} u_{*}|L| / Q$, and $x_{*}=x /|L|$. Equation 24, which can be used to estimate the ground-level impact of a line source (such as a road) can be rewritten as,

$$
\frac{\bar{C}^{y}}{Q}=\frac{1}{u_{*} x\left(1+\alpha(x /|L|)^{2}\right)^{1 / 2}} .
$$

Note that at small $x /|L|$, the crosswind integrated concentration depends only on $u_{*}$, which is relatively insensitive to errors in estimating the surface heat flux. At large $x /|L|$, the concentration depends on $u_{*}^{2} / Q_{o}$, and thus becomes more sensitive to both the surface friction velocity and the heat flux. This sensitivity to $Q_{o}$ is specific to Eq. 25; there are alternative expressions (see Nieuwstadt 1980) in which the concentration depends on $Q_{o}^{1 / 2}$ rather than $Q_{o}$.

Figure 8 compares estimates of $\bar{C}^{y} / Q$ based on $u_{*}$ and the heat flux estimated from Tillman's correction to the free convection formulation with those based on observed values of relevant micrometeorological parameters. The $95 \%$ confidence interval of the ratio of the observed to estimated $\bar{C}^{y} / Q$ at $x=10 \mathrm{~m}$ is only about 1.7 ; however, at $x=1,000 \mathrm{~m}$, the scatter is almost a factor of 4 .

This behaviour is readily explained. At small $x$ (Fig. 8a), the term $x /|L|$ in Eq. 25 plays a negligible role, and the concentration estimate is determined by $1 / u_{*}$. The scatter in the $\bar{C}^{y} / Q$ estimates about those based on observations reflects the errors in estimating $u_{*}$, shown 

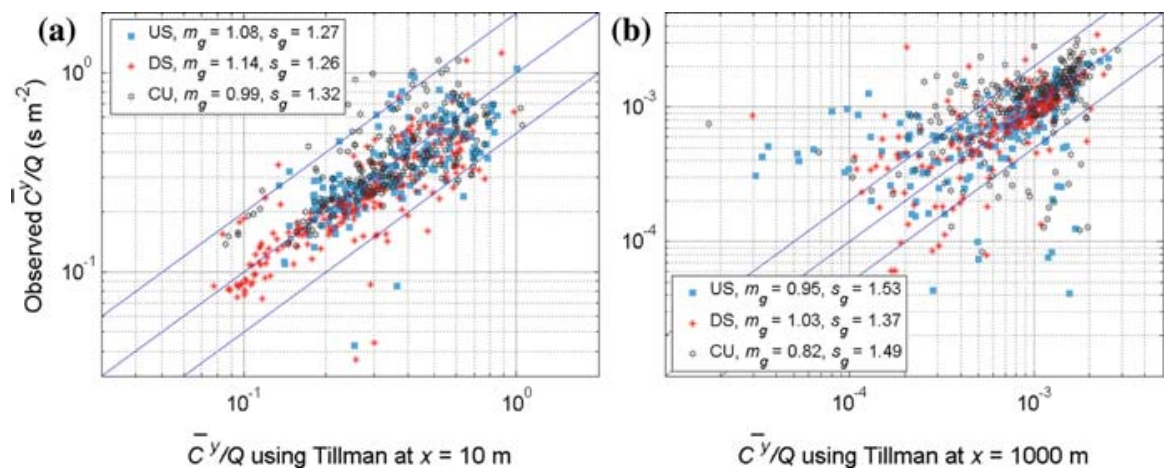

Fig. $8 \bar{C}^{y} / Q$ based on the estimated heat flux and $u_{*}$ from Tillman's method compared with those based on observed inputs for the US site (solid squares), DS site (stars), and CU site (open hexagrams) at a $x=10 \mathrm{~m}$ and $\mathbf{b} x=1,000 \mathrm{~m}$

in Fig. 7. At $1,000 \mathrm{~m}$ (Fig. 8b), the term $x /|L|$ becomes more important, and the scatter in the $\bar{C}^{y} / Q$ estimates is determined by the scatter in the estimated $u_{*}^{2} / Q_{o}$. Most of the lower values of $\bar{C}^{y} / Q$ are underestimated because $u_{*}$ is underestimated while the corresponding heat flux is overestimated. There are fewer overestimated points because the effect of overestimated $u_{*}$ is reduced by the overestimation of heat flux.

The uncertainty in estimating meteorological inputs can have a greater impact on concentrations from point sources because the horizontal plume spread of the point source plume is also affected by errors in estimating turbulent velocities. We examine this issue by modifying Eq. 25 to incorporate crosswind plume spread, $\sigma_{y}$, viz.

$$
\frac{C}{Q} \cong \frac{1}{\sigma_{y} u_{*} x\left[1+\alpha(x /|L|)^{2}\right]^{1 / 2}},
$$

where $\sigma_{y}$ is computed using $\sigma_{y} \cong \sigma_{v} x / u$, and $\sigma_{v}$ is estimated from Eq. 12, where $u$ is the value measured at the tower level of $3 \mathrm{~m}$.

Figure 9 shows estimates of $C / Q$ for $x=10 \mathrm{~m}$ (a) and $x=1,000 \mathrm{~m}$ (b) based on Tillman's method for heat flux plotted against those based on the observed values of $u_{*}$ and $L$. As expected, the scatter in the $C / Q$ estimates is larger than that for $\bar{C}^{y} / Q$ in Fig. 8. The comparison of Fig. $9 \mathrm{a}$ and $\mathrm{b}$ shows that the scatter in the concentration estimates increases with receptor distance. At $x=1,000 \mathrm{~m}$, the scatter in the $C / Q$ estimates is determined by the scatter in $u_{*}^{2} /\left(Q_{o} \sigma_{v}\right)$. Most of the low values of $C / Q$ are underestimated, which is similar to the underestimation of $\bar{C}^{y} / Q$ in Fig. 8. Model performance is similar for all the three methods of estimating heat flux (not shown).

\section{Comparison with the Surface Energy Balance Method}

This section examines whether measurements of the temperature fluctuations, $\sigma_{T}$ can reduce the uncertainty in energy balance methods used to estimate micrometeorological variables required for dispersion calculations. Computing the components of the energy balance at the surface requires information on cloud cover, albedo, and surface temperature to estimate the incoming and outgoing solar and thermal radiation fluxes. Because such information was not available, we used radiation measurements made at the US site during daytime (from 0900 to 

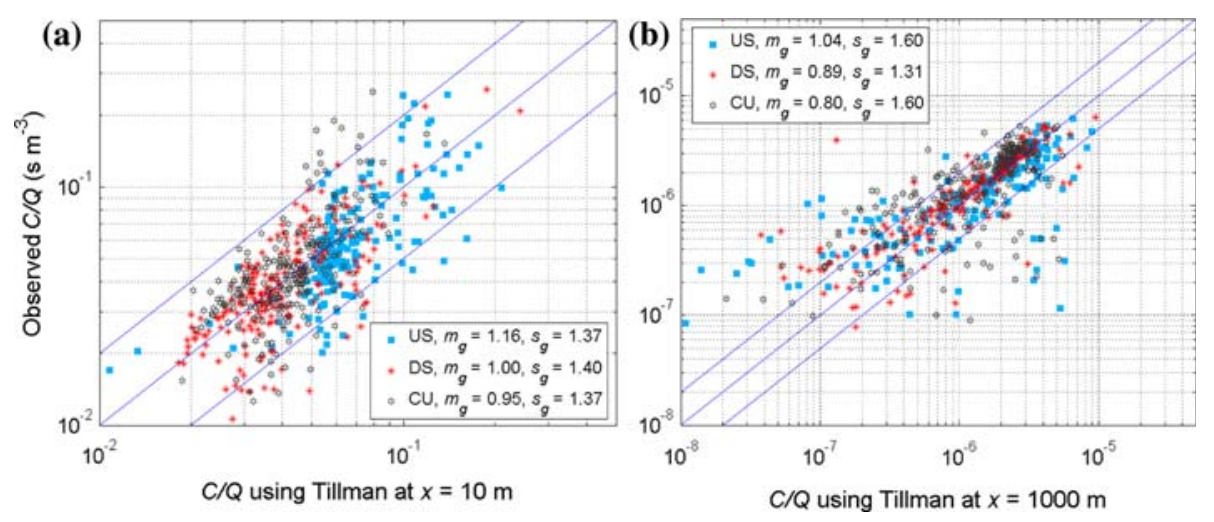

Fig. $9 C / Q$ based on the estimated heat flux and $u_{*}$ from Tillman's method compared with those based on observed inputs for the US site (solid squares), DS site (stars), and CU site (open hexagrams) at a $x=10 \mathrm{~m}$ and $\mathbf{b} x=1,000 \mathrm{~m}$

1700) when net radiation, $Q_{*}$, was positive. Note that using the observed net radiation instead of estimates based on cloud cover and albedo reduces some of the uncertainties in the energy balance method. The sensible heat flux $(H)$ was computed from the energy balance equation incorporated in meteorological processors typical of the current generation of dispersion models, such as AERMOD (Cimorelli et al. 2005); it is estimated from

$$
H=\frac{0.9 Q_{*}}{(1+1 / B o)},
$$

where $B o$, the Bowen ratio, is the ratio of the sensible to the latent heat flux. Here $H=Q_{0} \rho c_{p}$, where $\rho$ is the air density, and $c_{p}$ is the specific heat of air at constant pressure. The value of the Bowen ratio is highly uncertain because it depends on the moisture history of the soil. We took $B o=1.5$ by calibrating Eq. 27 with the maximum observed heat flux. Note that using a constant $B o$ cannot be readily justified because it depends on soil moisture availability, which is a function of time. Figure 10 compares the sensible heat-flux estimates from the energy balance method (Fig. 10a) with those from Eq. 22, and shows that estimates of heat flux based on $\sigma_{T}$ compare better with the observations than those derived from the surface energy balance method. The $95 \%$ confidence interval is reduced from about 2.9 (Fig. 10a) to about 1.7 (Fig. 10b).

Figure 11 compares estimates of $u_{*}$ with observations for the US site corresponding to the heat-flux estimates from Fig. 10. As indicated earlier, variations in the heat-flux estimates have little impact on estimates of $u_{*}$. Furthermore, these variations translate into less than noticeable differences in the estimates of $\sigma_{w}$ and $\sigma_{v}$ during daytime unstable conditions.

To examine the impact of differences in the heat-flux estimates on concentration calculations, we compared the computed concentrations at $1,000 \mathrm{~m}$ where stability effects become apparent through the term $x /|L|$ in Eq. 25. We see that, although the scatter is large for both methods of calculating heat flux, $s_{g}$ has a value of 1.5 for $\bar{C}^{y} / Q$ estimates when the heat flux is based on $\sigma_{T}$ (Fig. 12b), while $s_{g}$ is 2.2 for $\bar{C}^{y} / Q$ when the surface energy balance method is used to calculate heat flux (Fig. 12a). 
(a)

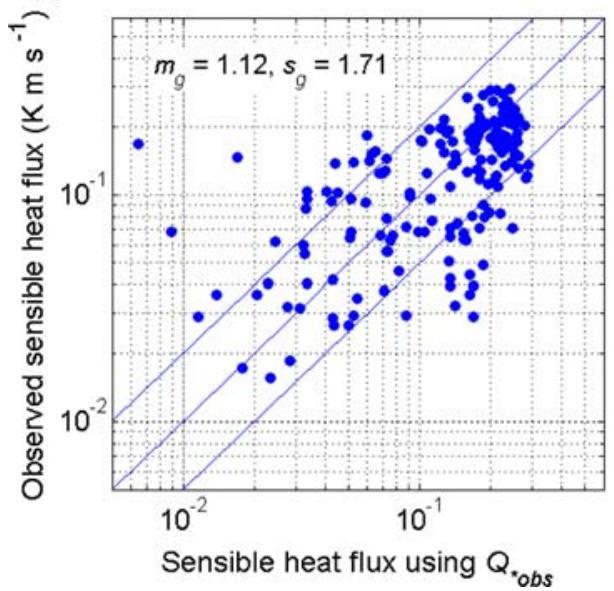

(b)

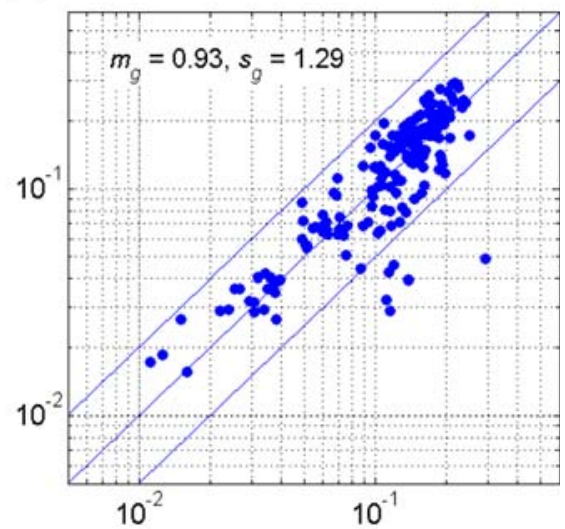

Sensible heat flux using Tillman

Fig. 10 Comparison of the sensible heat flux estimated from a the observed net radiation (Eq. 27), and b Tillman's method (Eq. 22) with those based on observed inputs for the US site during daytime unstable conditions
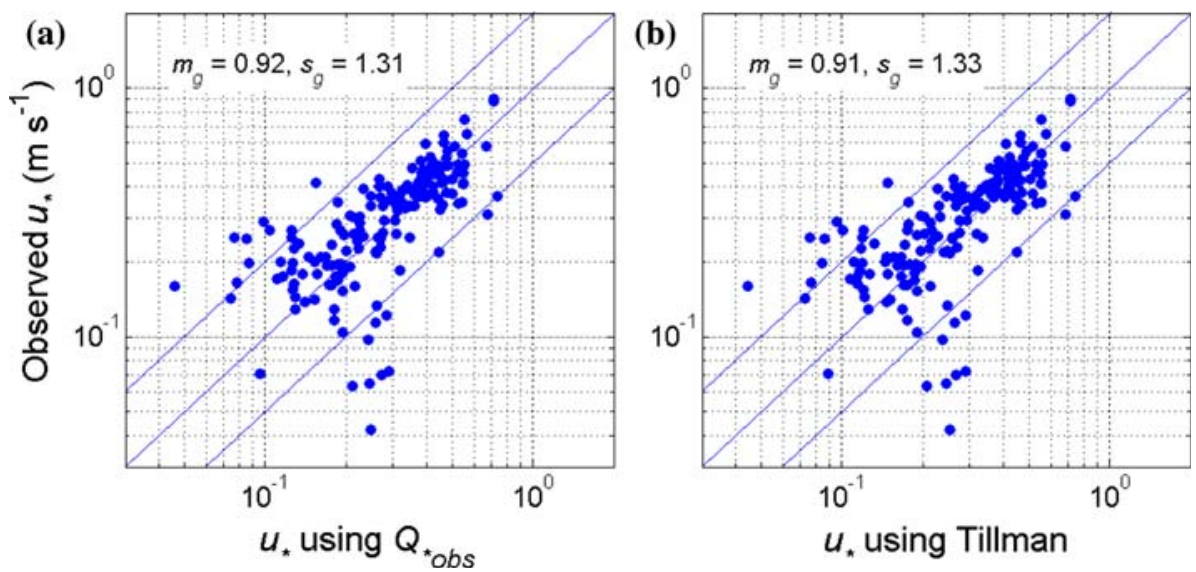

Fig. 11 Comparison of $u_{*}$ estimated from Eq. 5 with observations for the US site during daytime unstable conditions. Heat flux is estimated from a the observed net radiation (Eq. 27), and b Tillman's method (Eq. 22)

\section{Conclusions}

The results from this study show that measurements of wind speed and the standard deviation of temperature fluctuations at one level yield useful estimates of parameters required to model dispersion in both suburban and urban areas. Under unstable conditions, estimates of heat flux based on the measured $\sigma_{T}$ and wind speed at one level provide unbiased estimates of the surface friction velocity and turbulent velocities. The $95 \%$ confidence interval for the ratio of the observed and estimated value is about $1.7,1.4$ and 1.5 for $u_{*}, \sigma_{w}$ and $\sigma_{v}$ respectively. However, the ability to estimate micrometeorological variables is crucially dependent on adequate estimates of the aerodynamic roughness length at the site of interest. We suggest using empirical methods, such as that described herein, to estimate the aerodynamic rough- 
(a)

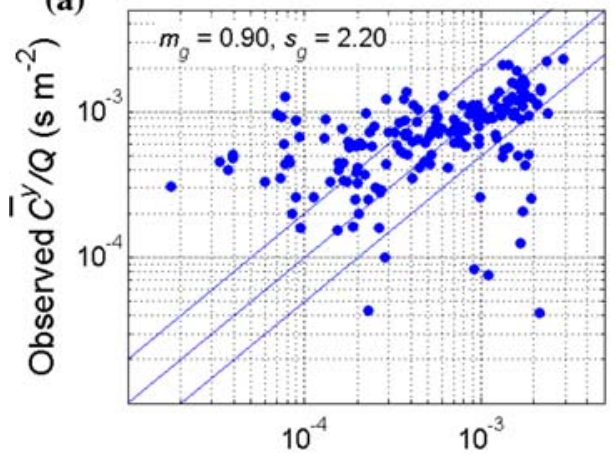

$\bar{C}^{y} / Q$ at $x=1000 \mathrm{~m}$ using $Q_{\text {*obs }}$ (b)

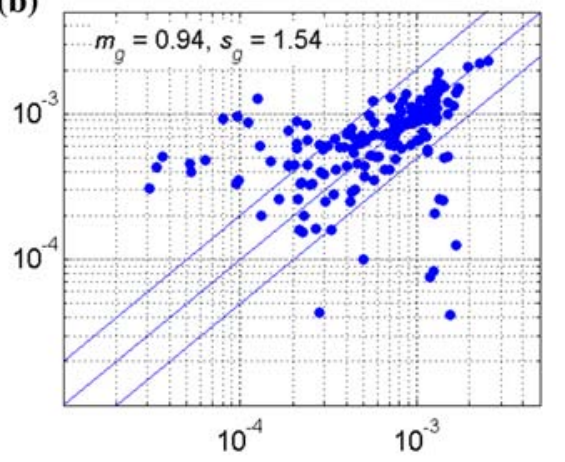

$\bar{C}^{y} / Q$ at $x=1000 \mathrm{~m}$ using Tillman

Fig. $12 \bar{C}^{y} / Q$ based on the estimated heat flux and $u_{*}$ from a the observed net radiation (Eq. 27), and b Tillman's method (Eq. 22) with those based on observed inputs for the US site and $x=1,000 \mathrm{~m}$ during daytime unstable conditions

ness length, although such methods have an inherent uncertainty that reflect the complexities of an urban area.

We examined two methods to account for shear effects on heat-flux estimates: one proposed by Tillman (1972) and the other based on a constant value of the correlation coefficient between the temperature and vertical velocity fluctuations. The results show that Tillman's method is superior to the free convection equation, which neglects shear effects. The scatter in the $u_{*}$ and heat-flux estimates leads to inevitable scatter in concentration estimates for near-surface line and point sources, although the impact is less for small downwind distances relative to the Obukhov length. The scatter in the concentration estimates for a point source is larger than that for a line source, because of the additional scatter introduced by errors in estimating the horizontal turbulent velocity used to compute horizontal plume spread.

The results indicate that measurements of $\sigma_{T}$ in addition to wind speed can reduce the uncertainty when using the energy balance method to estimate micrometeorological variables required to apply dispersion models in urban areas. Note that the energy balance method has been optimised using net radiation measurements and a calibrated value of the Bowen ratio. Even if radiation measurements are available, the energy balance method suffers from the need for an appropriate Bowen ratio (in addition to a roughness length) that can vary substantially both spatially as well as temporally (Ching 1985; Roth and Oke 1995).

Acknowledgements The research was sponsored by the National Science Foundation under grant ATMOS 0430776 and the California Energy Commission.

Open Access This article is distributed under the terms of the Creative Commons Attribution Noncommercial License which permits any noncommercial use, distribution, and reproduction in any medium, provided the original author(s) and source are credited.

\section{References}

Albertson JD, Parlange MB, Katul GG, Chu CR, Stricker H, Tyler S (1995) Sensible heat flux from arid regions using a simple flux-variance method. Water Resour Res 31:969-973 
Barad ML (ed) (1958) Project Prairie Grass. A field program in diffusion, Geophysical Research Paper No. 59, vols I (300 pp) and II (221 pp), AFCRF-TR-58-235, Air Force Cambridge Research Center, Bedford, MA

Britter RE, Hanna SR (2003) Flow and dispersion in urban areas. Annu Rev Fluid Mech 35:469-496

Businger JA (1973) Turbulent transfer in the atmospheric surface layer. In: Haugen DH (ed) Workshop on Micrometerology. American Meteorological Society, Boston, MA pp 67-100

Camuffo D, Bernardi A (1982) An observational study of heat fluxes and their relationship with net radiation. Boundary-Layer Meteorol 23:359-368

Carson DJ (1973) The development of a dry inversion-capped convectively unstable boundary layer. Q J Roy Meteorol Soc 99:450-467

Chang JC, Hanna SR (2004) Air quality model performance evaluation. Meteorol Atmos Phys 87:167-196

Ching JKS (1985) Urban-scale variations of turbulence parameters and fluxes. Boundary-Layer Meteorol $33: 335-361$

Christen A (2005) Atmospheric turbulence and surface energy exchange in urban environments. PhD Thesis in Meteorology. Institute of Meteorology, Climatology and Remote Sensing, University of Basel, $142 \mathrm{pp}$

Cimorelli AJ, Perry SG, Venkatram A, Weil JC, Paine RJ, Wilson RB, Lee RF, Peters WD, Brode RW (2005) AERMOD: a dispersion model for industrial source applications. Part I: general model formulation and boundary layer characterization. J Appl Meteorol 44:682-693

Clarke JF, Ching JKS, Godowitch JM (1982) An experimental study of turbulence in an urban environment. Tech. Rep. EPA 600/3-82-062, U.S. Environmental Protection Agency, Research Triangle Park, NC, $150 \mathrm{pp}$

De Bruin HAR, Kohsiek W, Van Den Hurk BJJM (1993) A verification of some methods to determine the fluxes of momentum, sensible heat and water vapour using standard deviation and structure parameter of scalar meteorological quantities. Boundary-Layer Meteorol 63:231-257

Feigenwinter C (2000) The vertical structure of turbulence above an urban canopy. $\mathrm{PhD}$ thesis, Institue of Meteorology, Climatology and Remote Sensing, University of Basel, 84 pp

Garratt JR (1980) Surface influence upon vertical profiles in the atmospheric near-surface layer. Q J Roy Meteorol Soc 106:803-819

Garratt JR (1983) Surface influence upon vertical profiles in the nocturnal boundary layer. Boundary-Layer Meteorol 26:69-80

Garratt JR (1992) The atmospheric boundary layer. Cambridge University Press, U.K., 316 pp

Grimmond CSB, Oke TR (1999a) Heat storage in urban areas: local-scale observations and evaluation of a simple model. J Appl Meteorol 38:922-940

Grimmond CSB, Oke TR (1999b) Aerodynamic properties of urban areas derived from analysis of surface form. J Appl Meteorol 38:1261-1292

Grimmond CSB, Cleugh HA, Oke TR (1991) An objective urban heat storage model and its comparison with other schemes. Atmos Environ 25:311-326

Hanna SR, Chang JC (1992) Boundary layer parameterizations for applied dispersion modeling over urban areas. Boundary-Layer Meteorol 58:229-259

Harman IN, Finnigan JJ (2007) A simple unified theory for flow in the canopy and roughness sublayer. Boundary-Layer Meteorol 123:339-363

Hicks B (1981) An examination of turbulence statistics in the surface boundary layer. Boundary-Layer Meteorol 21:389-402

Holtslag AAM, Ulden AP (1983) A simple scheme for daytime estimates of the surface fluxes from routine weather data. J Clim Appl Meteorol 22:517-529

Hsieh CI, Katul GG, Scheildge J, Sigmon JT, Knoerr KR (1996) Estimation of momentum and heat fluxes using dissipation and flux-variance methods in the unstable surface layer. Water Resour Res 8:2453-2462

Kaimal JC, Finnigan JJ (1994) Atmospheric boundary layer flows, their structure and measurement. Oxford University Press, New York, 289 pp

Kastner-Klein P, Rotach MW (2004) Mean flow and turbulence characteristics in an urban roughenss sublayer. Boundary-Layer Meteorol 111:55-84

Lloyd CR, Culf AD, Doman AJ, Gash JH (1991) Estimates of sensible heat flux from observations of temperature fluctuations. Boundary-Layer Meteorol 57:311-322

Luhar A, Venkatram A, Lee SM (2006) On relationships between urban and rural near-surface meteorology for diffusion applications. Atmos Environ 40:6541-6553

Monin AS, Yaglom AM (1971) Statistical fluid mechanics: mechanics of turbulence, vol 1. MIT Press, Cambridge, MA, 769 pp

Monteith JL (1981) Evaporation and surface temperature. Q J Roy Meteorol Soc 107:1-27

Nieuwstadt FTM (1980) Application of mixed-layer similarity to the observed dispersion from a ground-level source. J Appl Meteorol 19:157-162 
Oikawa S, Meng Y (1995) Turbulence characteristics and organized motion in a suburban roughness sublayer. Boundary-Layer Meteorol 74:289-312

Panofsky HA, Tennekes H, Lenschow DH, Wyngaard JC (1977) The characteristics of turbulent velocity components in the surface layer under convective conditions. Boundary-Layer Meteorol 11:355-359

Princevac M, Venkatram A (2007) Estimating micrometeorological inputs for modeling dispersion in urban areas during stable conditions. Atmos Environ 41:5345-5356

Raupach MR, Antonia RA, Rajagopalan S (1991) Rough-wall turbulent boundary layers. Appl Mech Rev 44:1-25

Rotach MW (1993) Turbulence close to a rough urban surface, Part II: variances and gradients. BoundaryLayer Meteorol 66:75-92

Rotach MW (1999) On the influence of the urban roughness sublayer on turbulence and dispersion. Atmos Environ 33:4001-4008

Roth M (1993) Turbulent transfer relationships over an urban surface. II. Integral statistics. Q J Roy Meteorol Soc 119:1105-1120

Roth M, Oke TR (1995) Relative efficiencies of turbulent transfer of heat, mass and momentum over a patchy urban surface. J Atmos Sci 52:1863-1874

Tillman JE (1972) The indirect determination of stability, heat and momentum fluxes in the atmospheric boundary layer from simple scalar variables during dry unstable conditions. J Appl Meteorol 11:783792

van Ulden AP, Holtslag AAM (1985) Estimation of atmospheric boundary layer parameters for diffusion applications. J Clim Appl Meteorol 24:1196-1207

Venkatram A (1992) Vertical dispersion of ground-level releases in the surface boundary layer. Atmos Environ 26:947-949

Venkatram A (2008) Computing and displaying model performance statistics. Atmos Environ 24:6862-6868

Venkatram A, Princevac M (2008) Using measurements in urban areas to estimate turbulent velocities for modeling dispersion. Atmos Environ 42:3833-3841

Wang IT, Chen PC (1980) Estimation of heat and momentum fluxes near the ground. In: Proceedings of 2nd joint conference on applications on air pollution. American Meteorological Society, 45 Beacon St., Boston, MA 02108, pp 764-769

Weaver HL (1990) Temperature and humidity flux-variance relations determined by one-dimensional eddy correlation. Boundary-Layer Meteorol 53:77-91

Wesely ML (1988) Use of variance techniques to measure dry air-surface exchange rates. Boundary-Layer Meteorol 44:13-31

Wieringa J (1993) Representative roughness parameters for homogeneous terrain. Boundary-Layer Meteorol 63:323-393 